\title{
Heat and Flow Characteristics of Nanofluid Flow in Porous Microchannels
}

\author{
T. W. Ting ${ }^{1 *}$, Y. M. Hung ${ }^{2}$, M. S. Osman ${ }^{1}$ and P. N. Y. Yek ${ }^{1}$ \\ ${ }^{1}$ School of Engineering and Technology, University College of Technology Sarawak, \\ 96000 Sibu, Sarawak, Malaysia \\ Email: dr.ting@ucts.edu.my \\ Phone: +6084367540; Fax: +6084367301 \\ ${ }^{2}$ School of Engineering, Monash University, \\ 47500 Bandar Sunway, Malaysia
}

\begin{abstract}
In the present study, convective flow of nanofluid in porous microchannel is analyzed by utilizing field synergy principle. The effects of porous medium embedment on the field synergy of water- $\mathrm{Al}_{2} \mathrm{O}_{3}$ nanofluid is investigated based on locally thermal nonequilibrium model. Energy equations for both fluid and solid phases are solved analytical while the field synergy formulations based on viscous dissipative flow are developed. It is observed that the interstitial heat transfer affects the field synergy of the flow tremendously. The deviation between one-energy-equation model and two-energyequation model reduces when the field synergy of the system increases. With the embedment of porous medium, the convection performance of microchannel is enhanced due to the increased synergy between the heat and flow fields. Besides, the field synergy of the system can be further enhanced by suspending nanoparticle in conventional fluid. This study provides an auxiliary point of view on the distinctive convection performance of nanofluid flow in porous microchannel.
\end{abstract}

Keywords: Field synergy; porous microchannel; nanofluid; viscous dissipation.

\section{INTRODUCTION}

Convection performance enhancement has been an everlasting issue attributable to the increasing demand of compact heat exchanger with high efficiency to reduce cost and energy consumption. Various techniques have been adopted for the enhancement of convective flow performance such as employing porous medium, duct rotation, fin attachment, twisted tape insert and vortex generator. However, up to the end of twentieth century, no unified theory can explain the underlying common essence of these enhancement techniques. In 1998, an analogy between convection and conduction with heat source has been developed by Guo et al. [1], leading to a novel theory which is denoted as field synergy principle [2]. Field synergy principle states that convection mechanism can be analyzed based on the synergetic relation between the heat and flow fields. The principle elucidates that the convection performance enhancements for parabolic flow [1] and elliptic flow [2] are ascribable to the improved synergy between the heat and flow fields. Zhu and Zhao [3] verified that the local convection performance of a laminar flow can be visualized through the distribution of synergy angle in the thermal boundary layer. It has been reported that the existing mechanisms for enhancing convection, such as decreasing thermal boundary layer, increasing flow 
interruption and increasing near-wall velocity gradient, can be unified under field synergy principle [4]. Therefore, field synergy principle appears to be a useful tool in enhancing the convective performance of thermal devices [5-11].

In the recent decades, micro-scale convection garners the attention of research community due to the formidable thermal management problem in the miniaturization of electronic devices. Microchannel heat sink appears to be a favorable solution owing to its large area-to-volume ratio which induces high heat transfer rate [12]. This ratio can be intensified through the embedment of porous medium [13] and hence enhances the heat removal rate to a greater extent [14]. Besides, convection performance can be further increased through the suspension of high-thermal-conductivity nanoparticle in conventional coolant [15-18]. This innovative type of fluid is coined as nanofluid which displays an exceptional potential in increasing the convection performance [19-22] because of the intensified synergy between the heat and flow fields [23-29].

In the existing literature, field synergy analysis of porous medium forced convection is extremely scarce and most of them utilized the locally thermal equilibrium model [30-32]. Field synergy analysis with two-energy-equation model has been used to analyze the convection performance of porous material adopted in concentrated solar power [33] and metal-foam duct [34]. However, none of these studies explicitly analyze the effects of porous medium embedment, i.e. permeability and interstitial heat transfer in affecting the synergy between the heat and flow fields, in spite of the fact that the these effects are consequential on the convection of a porous medium [35-37]. To bridge this information gap, field synergy principle is utilized in this study to analytically investigate the convection performance enhancement of nanofluid flow in porous microchannels. By considering the effects of interstitial heat transfer, twodimensional temperature distributions for both fluid and solid phases are obtained using the locally thermal non-equilibrium model. The field synergy formulations based on viscous dissipative flow are developed to scrutinize the synergetic relationship between the heat and flow fields of such integrated system. The effects of porous medium embedment, interstitial heat transfer and nanoparticle suspension on the field synergy of the system are presented, aiming to provide alternative point of view on the distinctive convection performance of viscous dissipative nanofluid flow in porous microchannel.

\section{MATHEMATICAL FORMULATION}

The schematic diagram of the present study is illustrated in Figure 1.

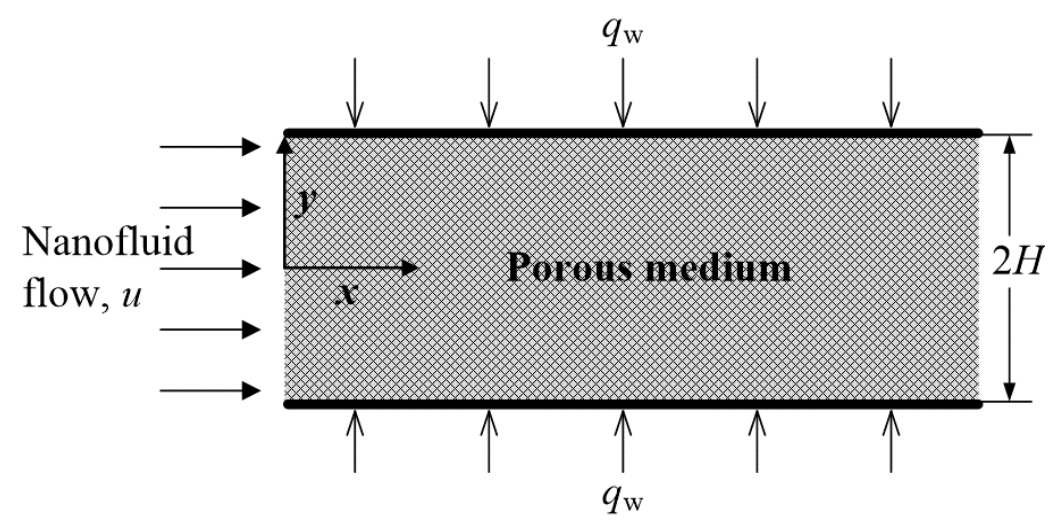

Figure 1. Schematic diagram of nanofluid convection in a parallel-plate microchannel. 
In this study, the convection of water- $\mathrm{Al}_{2} \mathrm{O}_{3}$ nanofluid in a parallel-plate microchannel embedded with silicon porous material is considered. A constant heat flux $q_{w}$ is imposed at the walls of the porous microchannel as shown in Figure 1. The thermal conductivity $k_{\mathrm{nf}}(\mathrm{W} / \mathrm{mK})$ of water- $\mathrm{Al}_{2} \mathrm{O}_{3}$ nanofluid and the thermal conductivity coefficient $C_{k}$ can be calculated from Eq. (1) and (2) respectively [38].

$$
\begin{aligned}
& k_{\mathrm{nf}}=C_{k} k_{\mathrm{f}} \\
& C_{k}=\frac{\left(1+40000 \operatorname{Re}_{\mathrm{b}}^{2.5} \operatorname{Pr}_{\mathrm{f}}^{1 / 3} \phi\right)\{\tau(1+2 \alpha)+2+2 \phi[\tau(1-\alpha)-1]\}}{\{\tau(1+2 \alpha)+2-\phi[\tau(1-\alpha)-1]\}}
\end{aligned}
$$

The parameter $\tau=k_{\mathrm{p}} / k_{\mathrm{f}}$ is the thermal conductivity ratio of nanoparticle thermal conductivity $k_{\mathrm{p}}(\mathrm{W} / \mathrm{mK})$ to base-fluid thermal conductivity $k_{\mathrm{f}}(\mathrm{W} / \mathrm{mK}), \operatorname{Pr}_{\mathrm{f}}=c_{p, \mathrm{f}} \mu_{\mathrm{f}} / k_{\mathrm{f}}$ is Prandtl number, $\phi$ is nanoparticle volume fraction, $\alpha=2 R_{\mathrm{b}} k_{\mathrm{f}} / d_{\mathrm{p}}$ is Biot number, where $d_{\mathrm{p}}(\mathrm{m})$ is nanoparticle diameter and $R_{\mathrm{b}}\left(\mathrm{m}^{2} \mathrm{~K} / \mathrm{W}\right)$ is interfacial resistance. BrownianReynolds number is expressed as $\operatorname{Re}_{\mathrm{b}}=\left(18 k_{\mathrm{b}} T / \pi \rho_{\mathrm{p}} d_{\mathrm{p}}\right)^{0.5} / \nu_{\mathrm{f}}$, with $k_{\mathrm{b}}(\mathrm{J} / \mathrm{K})$ as the Boltzmann constant and $v_{\mathrm{f}}\left(\mathrm{m}^{2} / \mathrm{s}\right)$ as the kinematic viscosity. For water- $\mathrm{Al}_{2} \mathrm{O}_{3}$ nanofluid, $R_{\mathrm{b}}=0.77 \times 10^{-8} \mathrm{~m}^{2} \mathrm{~K} / \mathrm{W}$ [38]. The effective viscosity of water- $\mathrm{Al}_{2} \mathrm{O}_{3}$ is defined as Eq. (3) [39].

$\mu_{\mathrm{nf}}=C_{\mu} \mu_{\mathrm{f}}$

In Eq.(3) $\mu_{\mathrm{f}}\left(\mathrm{Ns} / \mathrm{m}^{2}\right)$ is the dynamic viscosity of basefluid and the viscosity coefficient $C_{\mu}$ is given in Eq. (4).

$$
C_{\mu}=(1+2.5 \phi)\left[1+280\left(d_{p} \phi / D\right)^{1 / 2}\right]
$$

where $D(\mathrm{~m})$ is hydraulic diameter. The effective density and specific heat are, respectively, given by Eq. (5) and (6) [40].

$$
\begin{aligned}
& \rho_{\mathrm{nf}}=\rho_{\mathrm{f}}(1-\phi)+\rho_{\mathrm{p}} \phi \\
& c_{p, \mathrm{nf}}=\left[\rho_{\mathrm{f}} c_{p, \mathrm{f}}(1-\phi)+\rho_{\mathrm{p}} c_{p, \mathrm{p}} \phi\right] /\left[\rho_{\mathrm{f}}(1-\phi)+\rho_{\mathrm{p}} \phi\right]
\end{aligned}
$$

Based on Ting et al. [41], Brinkman momentum equation subjected to non-slip condition at channel walls and symmetrical condition at channel mid-plane can be solved analytically to yield Eq. (7).

$\hat{U}=u / \bar{u}=[S \cosh (S)-S \cosh (S Y)] /[S \cosh (S)-\sinh (S)]$

In Eq.(7), $u(\mathrm{~m} / \mathrm{s})$ is nanofluid velocity, $\bar{u}(\mathrm{~m} / \mathrm{s})$ is dimensional mean velocity over the channel cross-sectional area, $S=(M \mathrm{Da})^{-0.5}$ is porous medium shape factor, $M=$ $\mu_{\mathrm{eff}} / \mu_{\mathrm{nf}}$ is the viscosity ratio where $\mu_{\mathrm{eff}}\left(\mathrm{Ns} / \mathrm{m}^{2}\right)$ is porous medium effective viscosity, Da $=K / H^{2}$ is Darcy number with $K\left(\mathrm{~m}^{2}\right)$ as the permeability and $Y=y / H$. For the sake of 
brevity, it is assumed that $\mu_{\mathrm{eff}}=\mu_{\mathrm{nf}}$, yielding $M=1$ [42]. Based on the first-law formulation of Ting et al. [41], porous medium energy equations for both fluid and solid phases are, respectively, expressed as Eq. (8) and (9).

$$
\begin{aligned}
& k_{\mathrm{nf}, \mathrm{eff}} \frac{\partial^{2} T_{\mathrm{nf}}}{\partial y^{2}}+h_{i} a_{i}\left(T_{\mathrm{s}}-T_{\mathrm{nf}}\right)+\frac{\mu_{\mathrm{nf}} u^{2}}{K}+\mu_{\mathrm{eff}}\left(\frac{d u}{d y}\right)^{2}=\rho_{\mathrm{nf}} c_{p, \mathrm{nf}} u \frac{\partial T_{\mathrm{nf}}}{\partial x} \\
& k_{\mathrm{s}, \mathrm{eff}} \frac{\partial^{2} T_{\mathrm{s}}}{\partial y^{2}}-h_{i} a_{i}\left(T_{\mathrm{s}}-T_{\mathrm{nf}}\right)=0
\end{aligned}
$$

The effective conductivities for fluid and solid phases are, respectively, $k_{n f \text {,eff }}=$ $\varepsilon k_{n f}(\mathrm{~W} / \mathrm{mK})$ and $k_{s, \text { eff }}=(1-\varepsilon) k_{s}(\mathrm{~W} / \mathrm{mK})$. The parameter $\varepsilon$ is porous medium porosity, $h_{i}$ $\left(\mathrm{W} / \mathrm{m}^{2} \mathrm{~K}\right)$ is porous medium interstitial heat transfer coefficient and $a_{i}\left(\mathrm{~m}^{-1}\right)$ is porous medium specific surface area, $T(\mathrm{~K})$ is the temperature with the subscript s represents the solid phase while the subscript nf represents the fluid phase. A uniform wall heat flux $q_{\mathrm{w}}\left(\mathrm{W} / \mathrm{m}^{2}\right)$ is imposed at the microchannel walls and the symmetrical thermal boundary condition is applied at channel mid-plane, given by [35]:

$$
\begin{aligned}
& q_{\mathrm{w}}=\left.k_{\mathrm{s}, \mathrm{eff}} \frac{\partial T_{\mathrm{s}}}{\partial y}\right|_{y=H}+\left.k_{\mathrm{nf}, \mathrm{eff}} \frac{\partial T_{\mathrm{nf}}}{\partial y}\right|_{y=H} \\
& \left.\frac{\partial T_{\mathrm{s}}}{\partial y}\right|_{y=0}=\left.\frac{\partial T_{\mathrm{nf}}}{\partial y}\right|_{y=0}=0
\end{aligned}
$$

By solving Eq.(8) and Eq.(9) with the conditions stated in Eq.(10) and Eq.(11), the dimensionless-form of temperature distributions for both nanofluid and porous medium solid phase can be expressed as:

$$
\begin{aligned}
\theta_{\mathrm{nf}}(X, Y)= & 2 X\left(1+2(\kappa+1) \mathrm{Br}^{\prime} S^{2} F_{3}\right) / \kappa \operatorname{Re} \operatorname{Pr} \xi+\left(1-4 S^{2} / \mathrm{Bi}\right) F_{1} \cosh (2 S Y) \\
& +\left(1-S^{2} / \mathrm{Bi}\right) F_{2} \cosh (S Y)+F_{3}\left(Y^{2}-2 / \operatorname{Bi}(\kappa+1)\right) \\
& -\frac{F_{4}}{\kappa} \cosh \left(\sqrt{\operatorname{Bi}\left(\kappa^{-1}+1\right)} Y\right)+F_{5} \\
\theta_{\mathrm{s}}(X, Y)= & 2 X\left(1+2(\kappa+1) \mathrm{Br}^{\prime} S^{2} F_{3}\right) / \kappa \operatorname{Re} \operatorname{Pr} \xi+F_{1} \cosh (2 S Y)+F_{2} \cosh (S Y) \\
+ & F_{3}\left(Y^{2}+2 \kappa / \operatorname{Bi}(\kappa+1)\right)+F_{4} \cosh \left(\sqrt{\operatorname{Bi}\left(\kappa^{-1}+1\right)} Y\right)+F_{5}
\end{aligned}
$$

The dimensionless parameter $\theta=k_{s, \mathrm{eff}}\left(T-T_{\mathrm{w}, \text { in }}\right) / q_{\mathrm{w}} H$ where $T_{\mathrm{w}, \text { in }}(\mathrm{K})$ is wall inlet temperature. In Eq.(13), $X=x / L$ where $L(\mathrm{~m})$ is channel length, $\kappa=k_{n f \text {,eff }} / k_{s, \text { eff }}$ is thermal conductivity ratio, $\mathrm{Bi}=h_{\mathrm{i}} a_{\mathrm{i}} H^{2} / k_{s, \text { eff }}$ is Biot number, $\mathrm{Br}^{\prime}=\mu_{\mathrm{eff}} \bar{u}^{2} / q_{\mathrm{w}} H$ is Brinkman number, $\operatorname{Re}=2 H \rho_{\mathrm{nf}} \bar{u} / \mu_{\mathrm{eff}}$ is nanofluid Reynolds number, $\operatorname{Pr}=c_{p, \mathrm{nf}} \mu_{\mathrm{eff}} / k_{n f \text {,eff }}$ is nanofluid 
Prandtl number and $\xi=H / L$ is channel aspect ratio. The constants in Eq.(12) and Eq.(13) are defined by Eq. (14) to (18).

$$
\begin{aligned}
F_{1}= & -E_{1} \mathrm{Bi} / 4 S^{2}\left(-4 \kappa S^{2}+\kappa \mathrm{Bi}+\mathrm{Bi}\right) \\
F_{2}= & -E_{2} \mathrm{Bi} / S^{2}\left(-\kappa S^{2}+\kappa \mathrm{Bi}+\mathrm{Bi}\right) \\
F_{3}= & -E_{3} / 2(\kappa+1) \\
F_{4}= & \kappa\left\{\left[E_{1} \cosh (2 S) /\left(-4 \kappa S^{2}+\kappa \mathrm{Bi}+\mathrm{Bi}\right)\right]+\left[E_{2} \cosh (S) /\left(-\kappa S^{2}+\kappa \mathrm{Bi}+\mathrm{Bi}\right)\right]\right. \\
& \left.+\left[E_{3} / \mathrm{Bi}(\kappa+1)\right]\right\} \div(\kappa+1) \cosh \left(\sqrt{\mathrm{Bi}\left(\kappa^{-1}+1\right)}\right) \\
F_{5}= & \left(E_{1} \cosh (2 S)+4 E_{2} \cosh (S)+2 S^{2} E_{3}\right) / 4 S^{2}(\kappa+1)
\end{aligned}
$$

where

$$
\begin{aligned}
& E_{1}=\mathrm{Br}^{\prime} S^{2} E_{4}^{2} \\
& E_{2}=E_{4}-E_{1} \cosh (S) \\
& E_{3}=-E_{4} \cosh (S) \\
& E_{4}=S /[S \cosh (S)-\sinh (S)]
\end{aligned}
$$

For the characterization of convection rate between the channel wall and the fluid flow, Nusselt number can be expressed as Eq. (23).

$\mathrm{Nu}=\frac{2 H h}{k_{\mathrm{nf}, \mathrm{eff}}}=\frac{2 H q_{\mathrm{w}}}{k_{\mathrm{nf}, \mathrm{eff}}\left(T_{\mathrm{w}}-\bar{T}_{\mathrm{nf}}\right)}$

By using field synergy principle, the convective terms in Eq.(8) and Eq.(9) can be written in the form of dot product of velocity vector and temperature gradient vector. The intersection angle between these two vectors has to be reduced in order to increase the convection rate. By combining and integrating these two equations over the porous microchannel cross-sectional area, the vector form of energy equation can be written as:

$$
\rho_{\mathrm{nf}} c_{p, \mathrm{nf}} \int_{0}^{H}\left(\mathbf{V} \cdot \nabla T_{\mathrm{nf}}\right) d y=k_{\mathrm{s}, \mathrm{eff}} \int_{0}^{H} \nabla^{2} T_{\mathrm{s}} d y+k_{\mathrm{nf}, \mathrm{eff}} \int_{0}^{H} \nabla^{2} T_{\mathrm{nf}} d y+\frac{\mu_{\mathrm{nf}}}{K} \int_{0}^{H} u^{2} d y+\mu_{\mathrm{eff}} \int_{0}^{H}\left(\frac{d u}{d x}\right)^{2} d y
$$

By using the conditions stated in Eq.(10) and Eq.(11), Eq.(24) can be reduced to: 
$\rho_{\mathrm{nf}} c_{p, \mathrm{nf}} \int_{0}^{H}\left(\mathbf{V} \cdot \nabla T_{\mathrm{nf}}\right) d y=q_{\mathrm{w}}+\frac{\mu_{\mathrm{nf}}}{K} \int_{0}^{H} u^{2} d y+\mu_{\mathrm{eff}} \int_{0}^{H}\left(\frac{d u}{d x}\right)^{2} d y$

In Eq.(25), there are two different sources of heat fluxes, i.e. constant heat flux at channel wall and viscous heating of fluid flow. Therefore, the viscous heating term on the right-hand-side of the equation has to be incorporated in the convective heat transfer term on the left-hand-side of the equation [24]. For this purpose, an adiabatic condition is imposed at porous microchannel wall in Eq.(25) to get

$$
\rho_{\mathrm{nf}} c_{p, \mathrm{nf}} \int_{0}^{H}\left(\mathbf{V} \cdot \nabla T_{\mathrm{vd}}\right) d y=\frac{\mu_{\mathrm{nf}}}{K} \int_{0}^{H} u^{2} d y+\mu_{\mathrm{eff}} \int_{0}^{H}\left(\frac{d u}{d x}\right)^{2} d y
$$

The parameter $\nabla T_{\mathrm{vd}}(\mathrm{K} / \mathrm{m})$ is the vector of temperature gradient which is attributable to the viscous heating of fluid flow. Therefore, the convection term on the left-hand-side of Eq.(26) is equivalent to the viscous heating convection form. Eq.(26) can be substituted into Eq.(25) to yield Eq. (27).

$\rho_{\mathrm{nf}} c_{p, \mathrm{nf}} \int_{0}^{H}\left[\mathbf{V} \cdot\left(\nabla T_{\mathrm{nf}}-\nabla T_{\mathrm{vd}}\right)\right] d y=q_{\mathrm{w}}$

The dimensionless form of velocity and temperature gradient vectorial variables can be defined as:

$$
\mathbf{V}^{*}=\frac{\mathbf{V}}{\bar{u}}, \quad \nabla T^{*}=\frac{\nabla T}{\left(T_{\mathrm{w}}-\bar{T}_{\mathrm{nf}}\right) / H}
$$

The dimensionless vectors in Eq.(28) can be used for the non-dimensionalization of Eq.(27) to obtain:

$\operatorname{Pe} \int_{0}^{1}\left[\mathbf{V}^{*} \cdot\left(\nabla T_{\mathrm{nf}}^{*}-\nabla T_{\mathrm{vd}}^{*}\right)\right] d Y=\mathrm{Nu}$

The dot product of the convection term in Eq.(29) can be written as Eq. (30).

$$
\mathbf{V}^{*} \cdot\left(\nabla T_{\mathrm{nf}}^{*}-\nabla T_{\mathrm{vd}}^{*}\right)=\left|\mathbf{V}^{*}\right|\left|\nabla T_{\mathrm{nf}}^{*}-\nabla T_{\mathrm{vd}}^{*}\right| \cos \beta^{\prime}
$$

The parameter $\beta^{\prime}\left(^{\circ}\right)$ represents the synergy angle between the velocity and temperature gradient vectors. To achieve convection performance enhancement, synergy angle $\beta^{\prime}\left({ }^{\circ}\right)$ needs to be decreased in order to increase the left-hand-side term of Eq.(29). For the ideal case of $\cos \beta^{\prime}=1$, the heat and flow fields are at the highest degree of synergy. For a viscous dissipative flow, the synergy angle $\beta^{\prime}\left(^{\circ}\right)$ can be expressed as: 


$$
\beta^{\prime}=\cos ^{-1}\left(\frac{\mathbf{V}^{*} \cdot\left(\nabla T_{\mathrm{nf}}^{*}-\nabla T_{\mathrm{vd}}^{*}\right)}{\left|\mathbf{V}^{*}\right|\left|\nabla T_{\mathrm{nf}}^{*}-\nabla T_{\mathrm{vd}}^{*}\right|}\right)=\cos ^{-1}\left(\frac{\partial T_{\mathrm{nf}} / \partial x-\partial T_{\mathrm{vd}} / \partial x}{\sqrt{\left(\partial T_{\mathrm{nf}} / \partial x-\partial T_{\mathrm{vd}} / \partial x\right)^{2}+\left(\partial T_{\mathrm{nf}} / \partial y\right)^{2}}}\right)
$$

Equation (31) can be rendered dimensionless as Eq. (32).

$$
\beta^{\prime}=\cos ^{-1}\left(\frac{1}{\sqrt{1+(\kappa \mathrm{Pe} / 2)^{2}\left(\partial \theta_{\mathrm{nf}} / \partial Y\right)^{2}}}\right)
$$

The averaged synergy angle over the cross-sectional area of the microchannel can be expressed as:

$$
\bar{\beta}^{\prime}=\int_{0}^{1} \beta^{\prime} d Y
$$

For the sake of accuracy, Eq.(33) can be evaluated by employing Simpson's method. To characterize the degree of synergy between the heat and flow fields, a field synergy number Fc' can be derived from Eq.(29) to yield:

$$
\mathrm{Fc}^{\prime}=\int_{0}^{1}\left[\mathbf{V}^{*} \cdot\left(\nabla T_{\mathrm{nf}}^{*}-\nabla T_{\mathrm{vd}}^{*}\right)\right] d Y=\frac{\mathrm{Nu}}{\mathrm{Pe}}
$$

Equation (34) shows that Nusselt number, which characterizes the convective heat transfer performance, is proportional to the field synergy number of a flow.

\section{RESULTS AND DISCUSSION}

In the present study, the convection of water- $\mathrm{Al}_{2} \mathrm{O}_{3}$ nanofluid is confined to laminar flow regime. The input parameters used for the computations are shown in Table 1.

Table 1. The input parameters used for the computations in the present study.

\begin{tabular}{ll}
\hline Parameters & Values \\
\hline Peclet number, $C_{k}$ Pe & 100 \\
Darcy number, Da & 0.1 \\
Biot number, Bi & 0.05 \\
Wall heat flux, $q_{\mathrm{w}}$ & $5 \times 10^{3} \mathrm{~W} / \mathrm{m}^{2}$ \\
Wall temperature at the inlet, $T_{\mathrm{w}, \text { in }}$ & $300 \mathrm{~K}$ \\
Height of the microchannel, $2 H$ & $50 \mu \mathrm{m}$ \\
Length of the microchannel, $L$ & $0.03 \mathrm{~m}$ \\
Porosity of the porous material, $\varepsilon$ & 0.9 \\
Thermal conductivity of the porous material, $k_{\mathrm{s}}$ & $148 \mathrm{~W} / \mathrm{mK}$ \\
Diameter of the nanoparticle, $d_{\mathrm{p}}$ & $1 \mathrm{~nm}$ \\
Density of the nanoparticle, $\rho_{\mathrm{p}}$ & $3975 \mathrm{~kg} / \mathrm{m}^{3}$ \\
Thermal conductivity of the nanoparticle, $k_{\mathrm{p}}$ & $36 \mathrm{~W} / \mathrm{mK}$ \\
Specific heat capacity of the nanoparticle, $c_{p, \mathrm{p}}$ & $778.6 \mathrm{~J} / \mathrm{kgK}$ \\
Density of the base fluid, $\rho_{\mathrm{f}}$ & $997 \mathrm{~kg} / \mathrm{m}^{3}$
\end{tabular}


Thermal conductivity of the base fluid, $k_{\mathrm{f}}$

Dynamic viscosity of the base fluid, $\mu_{\mathrm{f}}$ Specific heat capacity of the base fluid, $c_{p, \mathrm{f}}$
$0.613 \mathrm{~W} / \mathrm{mK}$

$8.55 \times 10^{-4} \mathrm{Ns} / \mathrm{m}^{2}$ $4179 \mathrm{~J} / \mathrm{kgK}$

The local synergy angle $\beta^{\prime}$ of water- $\mathrm{Al}_{2} \mathrm{O}_{3}$ nanofluid along the transverse direction of the porous microchannel is depicted in Figure 2. At the mid-plane of the microchannel, the local synergy angle $\beta^{\prime}=0^{\circ}$ which implies that the synergy between the heat and flow fields is the optimal $\mathrm{Y}=0$. This is attributable to the zero transverse temperature gradient characteristic at the mid-plane of a fully developed flow as shown in Eq.(11). When furthering away from $\mathrm{Y}=0$, the intensity of field synergy decreases as $\beta^{\prime}$ increases, with the highest synergy angle located at $\mathrm{Y}= \pm 1$. By suspending $\mathrm{Al}_{2} \mathrm{O}_{3}$ nanoparticle in fluid, $\beta^{\prime}$ decreases throughout microchannel, indicating that the synergy between the heat and flow fields of the nanofluid flow is enhanced. The magnitude of field synergy enhancement increases with the increase of nanoparticle volume fraction. Previous studies [41, 43] have been reported that the presence of viscous heating deteriorates the convection performance of nanofluid flow due to the decrease of synergy between the heat and flow fields [24]. However, the suspension of nanoparticle can enhance the convection performance of porous microchannel when either the Reynolds number is low or the nanoparticle diameter is below the threshold value [41]. Based on Table 1, the Peclet number of the present study is small and the nanoparticle diameter is well below the threshold value of $20 \mathrm{~nm}$ [41]. Therefore, the convection performance enhancement of viscous dissipative nanofluid flow in porous microchannel is attributable to the increased synergy between the heat and flow fields signified by the decrease of synergy angle as illustrated in Figure 2.

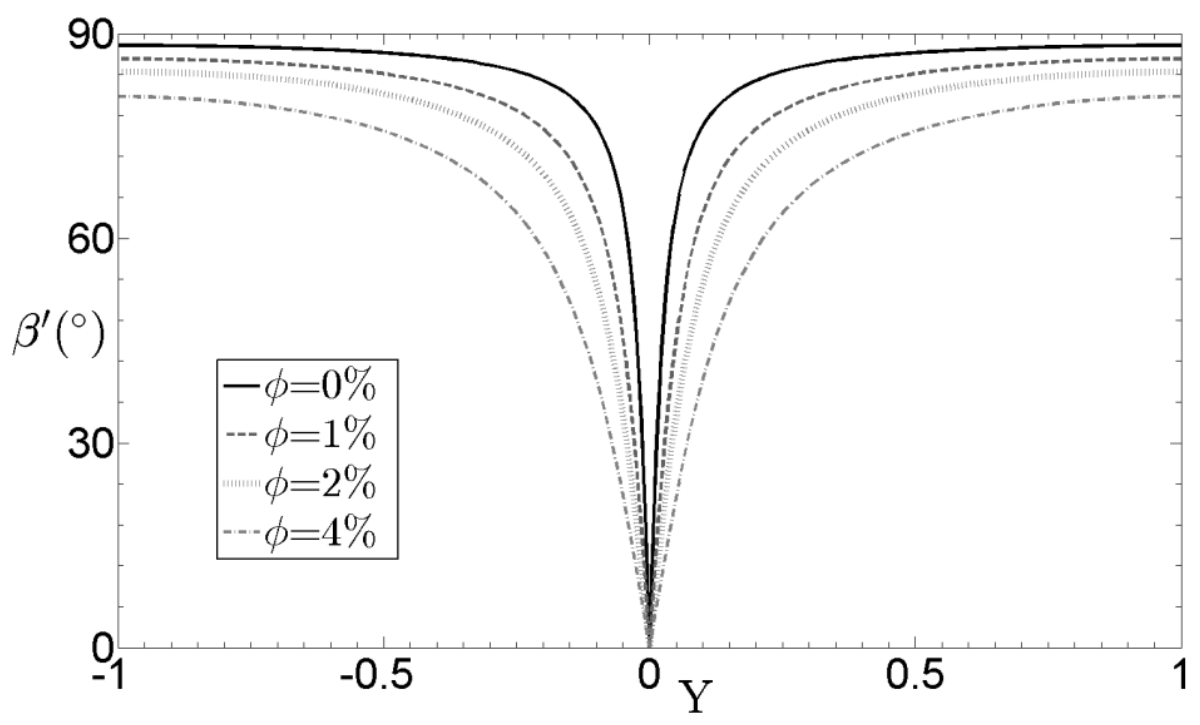

Figure 2. Transverse synergy angle $\beta^{\prime}$ of water- $\mathrm{Al}_{2} \mathrm{O}_{3}$ nanofluid with $\phi$ as a parameter.

The permeability of a porous medium can be characterized by Darcy number which is a crucial parameter in the convection analysis of a porous-medium flow. Therefore, it is imperative to scrutinize its effect on the convection performance of nanofluid flow from field synergy point of view. Figure 3 depicts the transverse synergy angle $\beta^{\prime}$ of water- $\mathrm{Al}_{2} \mathrm{O}_{3}$ nanofluid $(\phi=4 \%)$ with Darcy number as a parameter. With the reduction of Darcy number, $\beta^{\prime}$ decreases throughout the microchannel and therefore 
enhances the degree of field synergy. This is conceivable because the flow resembles the case of a clear-fluid flow at high Darcy number. With the embedment of porous medium, the convection performance is enhanced because of the intensification of synergy between the heat and flow fields. This can be justified by Figure 4 which shows that when the Darcy number decreases, the field synergy number Fc' increases, leading to the enhancement of synergy between the heat and flow fields and hence augment the convection performance in low-Darcy number regime. On the other hand, the suspension of nanoparticle in fluid further reduces $\beta^{\prime}$ and hence increases Fc' and convection performance of the flow.

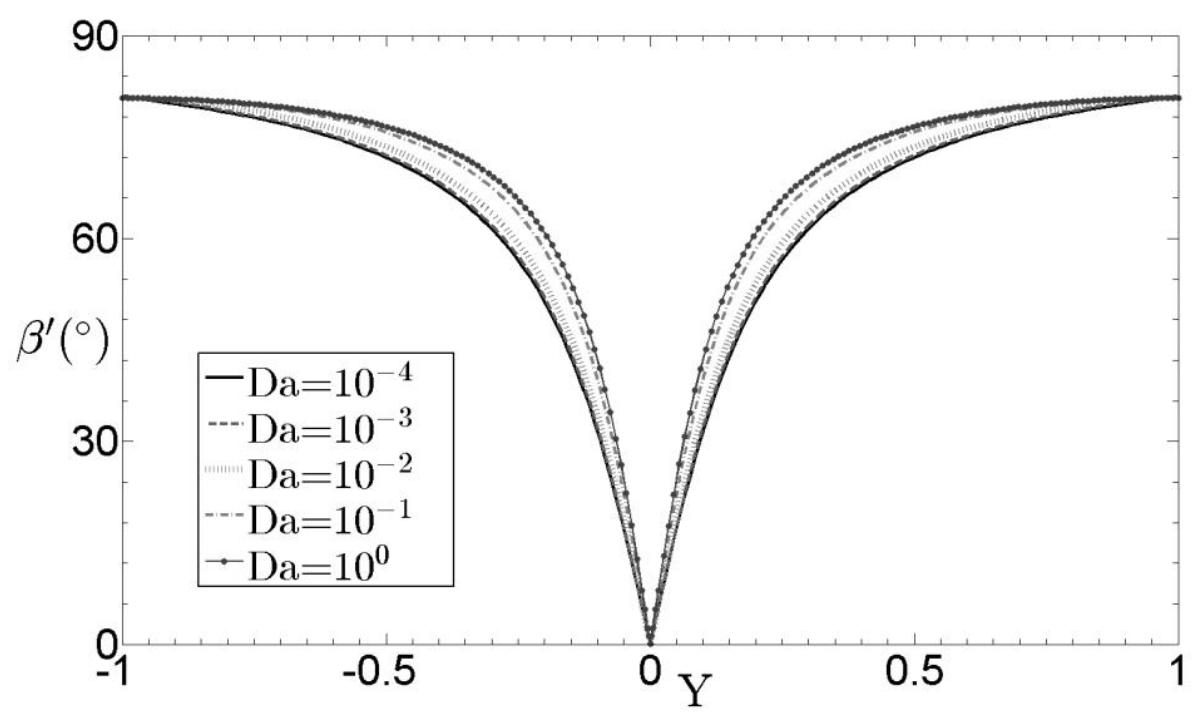

Figure 3. Transverse synergy angle $\beta^{\prime}$ of water- $\mathrm{Al}_{2} \mathrm{O}_{3}$ nanofluid with $\mathrm{Da}$ as a parameter.

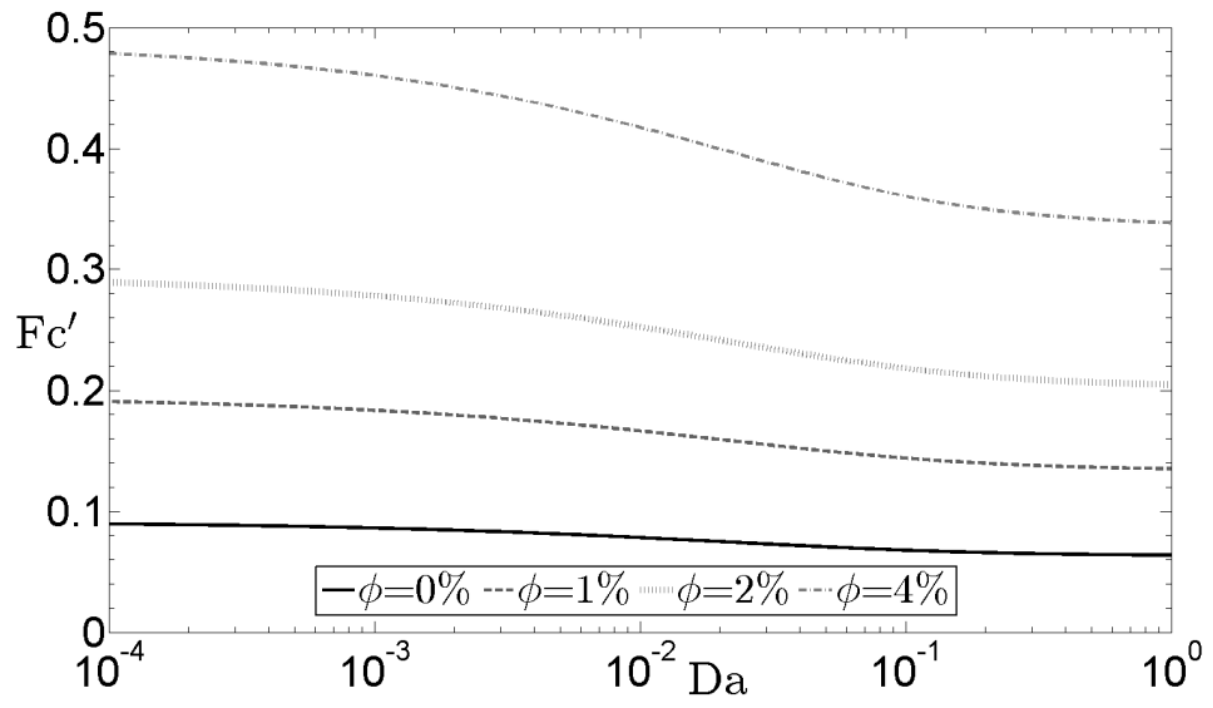

Figure 4. Variation of field synergy number Fc' as a function of Darcy number.

The variations of averaged synergy angle and field synergy number as a function of Biot number is illustrated in Figure 5. As Biot number increases, the interstitial heat transfer intensifies, causing the temperature gradient in nanofluid to increase and hence 
reduces the synergy angle along the transverse direction of the microchannel. Correspondingly, the increase of Biot number remarkably reduces the average synergy number and hence increases the field synergy number of the system as depicted in Figure 5. Correspondingly, the convection performance increases because of the enhanced field synergy of the flow. In the present analysis, the averaged synergy angle of water- $\mathrm{Al}_{2} \mathrm{O}_{3}$ nanofluid flow with $\phi=4 \%$ decreases as much as $44 \%$ while its field synergy number increases fourfold as Biot number increases from $10^{-2}$ to $10^{2}$. It has been reported that at high Biot number, the temperature difference between the two phases in porous medium is trivial and hence the two-energy-equation (locally thermal non-equilibrium) model can be approximated to the one-energy-equation (locally thermal equilibrium) model with negligible error [35]. From field synergy point of view, the deviation between these two models reduces when the level of synergy between the heat and flow fields increases.

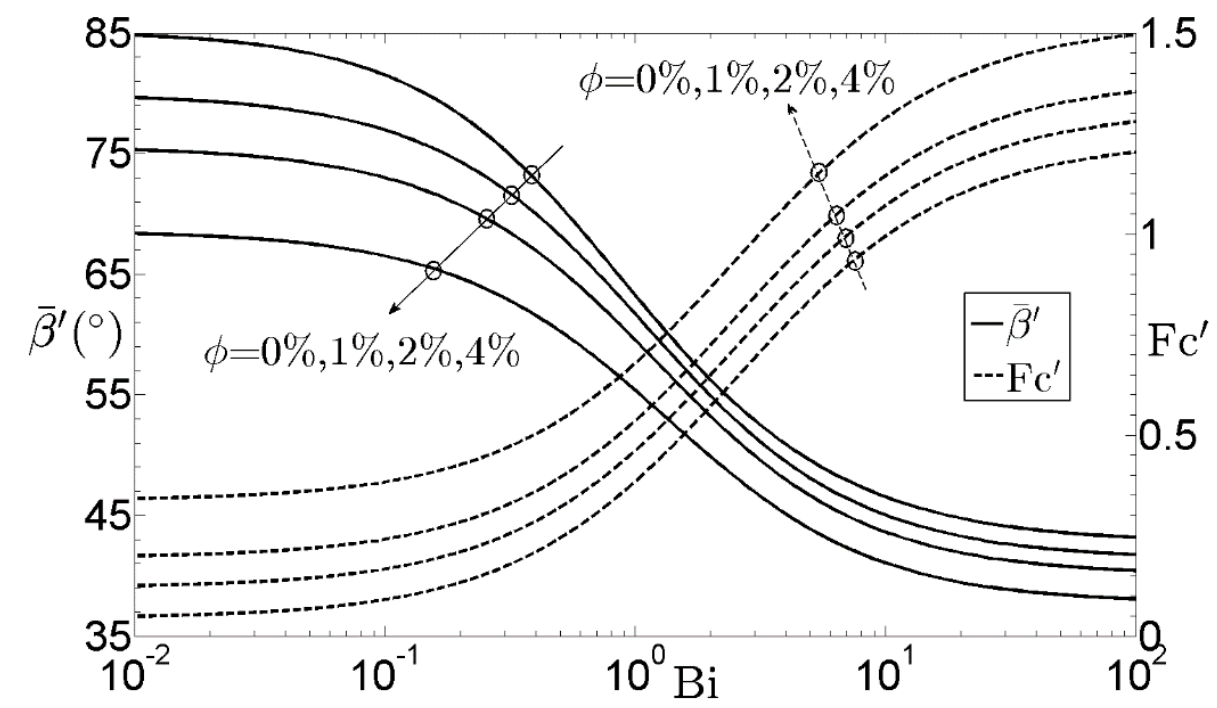

Figure 5. Variations of averaged synergy angle $\bar{\beta}^{\prime}$ and field synergy number Fc' as a function of Biot number.

\section{CONCLUSION}

From field synergy point of view, nanofluid convection in thermal non-equilibrium porous media embedded in microchannel is investigated. Two-dimensional temperature distribution for both fluid and solid phases are obtained. The field synergy formulations based on viscous dissipative flow are developed to study the synergy between the heat and flow fields of such integrated system. The embedment of porous media in microchannel deems to reduce synergy angle and increase field synergy number, which in turn augments the convection performance of the system. Besides, decreasing Darcy number and increasing Biot number can enhance the coordination between the heat and flow fields. The field synergy of the system can be further intensified by suspending nanoparticle in fluid. Besides, the discrepancy between the one-energy-equation model and two-energy-equation model is reduced when the field synergy of the system increases. 


\section{ACKNOWLEDGEMENT}

The authors would like to acknowledge University College of Technology Sarawak for supporting this work under University Research Grant (UCTS/RESEARCH/1/2016/05).

\section{REFERENCES}

[1] Guo ZY, Li DY, Wang BX. A novel concept for convective heat transfer enhancement. International Journal of Heat and Mass Transfer. 1998; 41:22212225.

[2] Tao WQ, Guo ZY, Wang BX. Field synergy principle for enhancing convective heat transfer - Its extension and numerical verifications. International Journal of Heat and Mass Transfer. 2002; 45:3849-3856.

[3] Zhu XW, Zhao JQ. Improvement in field synergy principle: More rigorous application, better results. International Journal of Heat and Mass Transfer. 2016; 100:347-354.

[4] Tao WQ, He YL, Wang QW, Qu ZG, Song FQ. A unified analysis on enhancing single phase convective heat transfer with field synergy principle. International Journal of Heat and Mass Transfer. 2002; 45:4871-4879.

[5] Guo J, Huai X. Numerical investigation of helically coiled tube from the viewpoint of field synergy principle. Applied Thermal Engineering. 2016; 98:137-143.

[6] Zhang B, Hamid MOA, Liu W. Numerical and experimental study of field synergy analysis in water jet impingement based on minimum entropy generation method. Applied Thermal Engineering. 2016; 99:944-958.

[7] Lu G, Zhou G. Numerical simulation on performances of plane and curved winglet - Pair vortex generators in a rectangular channel and field synergy analysis. International Journal of Thermal Sciences. 2016; 109:323-333.

[8] Zhang J, Tong L, Wang L. Field synergy characteristics in condensation heat transfer with non-condensable gas over a horizontal tube. AIP Advances. 2017; 7:055101.

[9] Deng Y, Cui J, Jiaqiang E, Zhang B, Zhao X, Zhang Z, Han D. Investigations on the temperature distribution of the diesel particulate filter in the thermal regeneration process and its field synergy analysis. Applied Thermal Engineering. 2017; 123:92-102.

[10] Yu ZQ, Wang P, Zhou WJ, Li ZY, Tao WQ. Study on the consistency between field synergy principle and entransy dissipation extremum principle. International Journal of Heat and Mass Transfer. 2018; 116:621-634.

[11] Mehra B, Simo Tala JV, Habchi C, Harion JL. Local field synergy analysis of conjugate heat transfer for different plane fin configurations. Applied Thermal Engineering. 2018; 130:1105-1120.

[12] Abdollahi A, Sharma RN, Vatani A. Fluid flow and heat transfer of liquid-liquid two phase flow in microchannels: A review. International Communications in Heat and Mass Transfer. 2017; 84:66-74.

[13] Kasaeian A, Daneshazarian R, Mahian O, Kolsi L, Chamkha AJ, Wongwises S, Pop I. Nanofluid flow and heat transfer in porous media: A review of the latest developments. International Journal of Heat and Mass Transfer. 2017; 107:778791. 
[14] Nojoomizadeh M, Karimipour A, Firouzi M, Afrand M. Investigation of permeability and porosity effects on the slip velocity and convection heat transfer rate of $\mathrm{Fe} 3 \mathrm{O} 4 /$ water nanofluid flow in a microchannel while its lower half filled by a porous medium. International Journal of Heat and Mass Transfer. 2018; 119:891-906.

[15] Yang L, Xu J, Du K, Zhang X. Recent developments on viscosity and thermal conductivity of nanofluids. Powder Technology. 2017; 317:348-369.

[16] Taherian H, Alvarado JL, Languri EM. Enhanced thermophysical properties of multiwalled carbon nanotubes based nanofluids. Part 1: Critical review. Renewable and Sustainable Energy Reviews. 2018; 82:4326-4336.

[17] Das PK. A review based on the effect and mechanism of thermal conductivity of normal nanofluids and hybrid nanofluids. Journal of Molecular Liquids. 2017; 240:420-446.

[18] Ganvir RB, Walke PV, Kriplani VM. Heat transfer characteristics in nanofluid A review. Renewable and Sustainable Energy Reviews. 2017; 75:451-460.

[19] Gupta M, Singh V, Kumar R, Said Z. A review on thermophysical properties of nanofluids and heat transfer applications. Renewable and Sustainable Energy Reviews. 2017; 74:638-670.

[20] Yang L, Du K. A comprehensive review on heat transfer characteristics of TiO2 nanofluids. International Journal of Heat and Mass Transfer. 2017; 108:11-31.

[21] Yazid MNAWM, Sidik NAC, Yahya WJ. Heat and mass transfer characteristics of carbon nanotube nanofluids: A review. Renewable and Sustainable Energy Reviews. 2017; 80:914-941.

[22] Gupta NK, Tiwari AK, Ghosh SK. Heat transfer mechanisms in heat pipes using nanofluids - A review. Experimental Thermal and Fluid Science. 2018; 90:84100.

[23] Yang J, Li F, Ni M, Yu B. Analysis of heat transfer performance for turbulent viscoelastic fluid-based nanofluid using field synergy principle. Science China Technological Sciences. 2015; 58:1137-1145.

[24] Ting TW, Hung YM, Guo N. Field-synergy analysis of viscous dissipative nanofluid flow in microchannels. International Journal of Heat and Mass Transfer. 2014; 73:483-491.

[25] Zhao N, Li S, Yang J. A review on nanofluids: Data-driven modeling of thermalphysical properties and the application in automotive radiator. Renewable and Sustainable Energy Reviews. 2016; 66:596-616.

[26] Zakaria I, Michael Z, Mohamed WANW, Mamat AMI, Azmi WH, Mamat R, Saidur R. A review of nanofluid adoption in polymer electrolyte membrane (PEM) fuel cells as an alternative coolant. Journal of Mechanical Engineering and Sciences. 2015; 8:1351-1366.

[27] Hamid KA, Azmi WH, Mamat R, Usri NA, Najafi G. Effect of temperature on heat transfer coefficient of titanium dioxide in ethylene glycol-based nanofluid. Journal of Mechanical Engineering and Sciences. 2015; 8:1367-1375.

[28] Abdullah A, Mohamad IS, Bani Hashim AY, Abdullah N, Wei PB, Isa MHM, Zainal Abidin S. Thermal conductivity and viscosity of deionised water and ethylene glycol-based nanofluids. Journal of Mechanical Engineering and Sciences. 2016; 10:2249-2261.

[29] Zainal Abidin S, Mohamad IS, Bani Hashim AY, Abdullah N, Hafiz MIM, Masripan NAB, Abdullah A. Investigation of thermal characteristics of CNF- 
based nanofluids for electronic cooling applications. Journal of Mechanical Engineering and Sciences. 2016; 10:2336-2349.

[30] Chen GM, Tso CP, Hung YM. Field synergy principle analysis on fully developed forced convection in porous medium with uniform heat generation. International Communications in Heat and Mass Transfer. 2011; 38:1247-1252.

[31] Chen GM, Tso CP. Field synergy principle analysis on convective heat transfer in porous medium with uniform heat generation for thermally developing flow. International Journal of Heat and Mass Transfer. 2012; 55:4139-4147.

[32] Wang B, Hong Y, Hou X, Xu Z, Wang P, Fang X, Ruan X. Numerical configuration design and investigation of heat transfer enhancement in pipes filled with gradient porous materials. Energy Conversion and Management. 2015; 105:206-215.

[33] Yu ZQ, Feng YL, Zhou WJ, Jin Y, Li MJ, Li ZY, Tao WQ. Study on flow and heat transfer characteristics of composite porous material and its performance analysis by FSP and EDEP. Applied Energy. 2013; 112:1367-1375.

[34] Xu H, Gong L, Huang S, Xu M. Flow and heat transfer characteristics of nanofluid flowing through metal foams. International Journal of Heat and Mass Transfer. 2015; 83:399-407.

[35] Lee DY, Vafai K. Analytical characterization and conceptual assessment of solid and fluid temperature differentials in porous media. International Journal of Heat and Mass Transfer. 1999; 42:423-435.

[36] Marafie A, Vafai K. Analysis of non-Darcian effects on temperature differentials in porous media. International Journal of Heat and Mass Transfer. 2001; 44:4401-4411.

[37] Chen GM, Tso CP. Forced convection with viscous dissipation using a twoequation model in a channel filled by a porous medium. International Journal of Heat and Mass Transfer. 2011; 54:1791-1804.

[38] Prasher R, Bhattacharya P, Phelan PE. Thermal conductivity of nanoscale colloidal solutions (nanofluids). Physical Review Letters. 2005; 94:025901.

[39] Jang SP, Lee JH, Hwang KS, Choi SUS. Particle concentration and tube size dependence of viscosities of $\mathrm{Al} 2 \mathrm{O} 3$-water nanofluids flowing through microand minitubes. Applied Physics Letters. 2007; 91:243112.

[40] Khanafer K, Vafai K. A critical synthesis of thermophysical characteristics of nanofluids. International Journal of Heat and Mass Transfer. 2011; 54:44104428.

[41] Ting TW, Hung YM, Guo N. Viscous dissipative forced convection in thermal non-equilibrium nanofluid-saturated porous media embedded in microchannels. International Communications in Heat and Mass Transfer. 2014; 57:309-318.

[42] Nield DA, Kuznetsov AV, Xiong M. Effects of viscous dissipation and flow work on forced convection in a channel filled by a saturated porous medium. Transport in Porous Media. 2004; 56:351-367.

[43] Mah WH, Hung YM, Guo N. Entropy generation of viscous dissipative nanofluid flow in microchannels. International Journal of Heat and Mass Transfer. 2012; 55:4169-4182. 